\title{
MicroRNA Let-7g and Atherosclerosis Plaque Stabilization
}

\author{
Rongping Yin 1,2, Chenlin Zhang2, Yunying Hou ${ }^{1}$, Xiaohua Wang $^{1 *}$ \\ ${ }^{1}$ The Division of Cardiology, The First Affiliated Hospital of Soochow University, Suzhou 215006, China \\ ${ }^{2}$ Cyrus Tang Hematology Center, Jiangsu Institute of Hematology, The Collaborative Innovation Center of Hematology, \\ Soochow University, Suzhou, China \\ Email: *sxwang2001@163.com
}

How to cite this paper: Yin, R.P., Zhang, C.L., Hou, Y.Y. and Wang, X.H. (2017) MicroRNA Let-7g and Atherosclerosis Plaque Stabilization. World Journal of Cardiovascular Diseases, 7, 24-36. https://doi.org/10.4236/wjcd.2017.72003

Received: December 30, 2016

Accepted: February 17, 2017

Published: February 20, 2017

Copyright ( $) 2017$ by authors and Scientific Research Publishing Inc. This work is licensed under the Creative Commons Attribution International License (CC BY 4.0).

http://creativecommons.org/licenses/by/4.0/ c) (i) Open Access

\begin{abstract}
Vascular atherosclerotic vulnerable plaque rupture is the primary cause of acute myocardial infarctions and strokes. Thus, stabilization of vulnerable plaques is of important clinical endeavor to decrease the fatal risk of atherosclerosis. Inflammatory infiltration, apoptosis of endothelial cells (ECs) and vascular smooth muscle cells (VSMCs), destruction of extracellular matrix (ECM) or collagen, and neovascularization all contribute to the formation and stability of plaque. Let-7g, one miRNA of let-7 family, is related to retardation of the progress of vulnerable atherosclerosis plaque. First of all, let-7g induced preservation on vascular diseases through regulating on the intracellular $\mathrm{Ca}^{2+}$ activated protein kinase C-oxLDL-LOX-1 pathway, which resulted in reduced leukocyte adhesion to and migration across endothelium. Over expression of let-7g negatively regulated apoptosis in the ECs by targeting lectin-like oxidized LDL receptor-1(LOX-1)/CASP3 expression, therefore made the fibrous cap of plaque integrated and thick, increased the density of vascular atherosclerotic plaque. In addition, let- $7 \mathrm{~g}$ might stabilize the atherosclerotic plaque through other aspects. In this review, we focus on current and potential importance of let-7g on the stabilization of atherosclerosis plaque which might lead to the future development of an alternative strategy of CAD.
\end{abstract}

\section{Keywords}

Atherosclerosis, Let-7g, MicroRNAs, Plaque, Stabilization

\section{Introduction}

Vascular atherosclerotic plaque rupture is the major cause of acute myocardial infarctions and strokes [1]. In clinical endeavor, stabilizing the vulnerable plaques is important to reduce the fatal risk of atherosclerosis [2]. Vulnerable pla- 
ques usually have thin, highly inflamed, and collagen-poor fibrous caps. Various intrinsic and extrinsic factors influence the stability of plaques, resulting in lifethreatening complications. Those factors include, but not limit to, continuous inflammatory responses, matrix degradation, and cell death or apoptosis. These changes lead to thinner fibrous cap and prone to rupture. In advanced atherosclerotic lesions, ectopic angiogenesis within the intima and media considered to be another vulnerable sign [3]. The number of neovessels spouted intraplaque indicates an aggravated plaque lesion because angiogenesis can be a source of intraplaque hemorrhage [4]. In short, the characteristics of unstable atherosclerosis plaque commonly include following aspects: the larger lipid nucleus of atherosclerosis plaque; the thinner fibrous cap; the less dense of vascular wall and plaque; and intraplaque ectopic angiogenesis (Figure 1(A)).

MicroRNAs (miRNAs), a kind of noncoding RNA, are one of the largest gene families [5]. They have been indicated intargeting around $60 \%$ of human genes and regulated a range of biological processes, such as TGF- $\beta$ and SIRT-1 signaling, Fas/FasL apoptotic pathways, and NF- $\kappa$ B pathway [6]. Moreover, miRNAs are associated with apoptosis, cell maturation, oxidative stress, degradation of extracellular matrix (ECM), angiogenesis, and inflammation [7] [8] [9] [10].

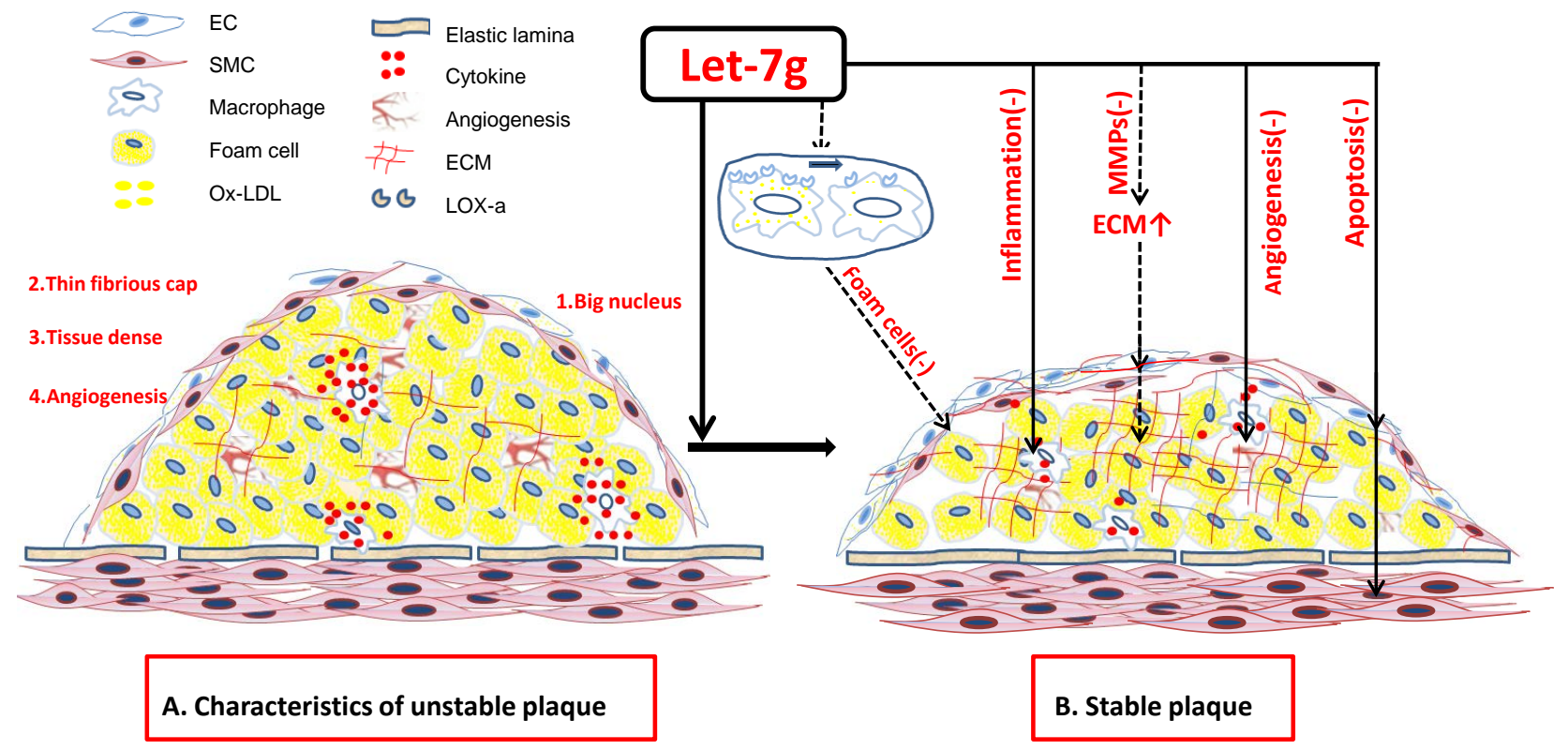

Figure 1. The current and hypothetic mechanisms of let-7g on the formation and stabilization of atherosclerosis plaque (A) The characteristics of unstable atherosclerosis plaque include four aspects: 1) the larger lipid nucleus of atherosclerosis plaque; 2) the thinner fibrous cap; 3) the less dense of vascular wall and plaque; and 4.intraplaque ectopic angiogenesis. (B) The current and hypothetic mechanisms of let-7g on the formation and stabilization of atherosclerosis plaque. Inflammation(-): let-7g could inhibit the inflammatory reaction of plaque and vessel wall. Apoptosis(-): let-7g could increase the thickness of the fibrous cap through restraining the apoptosis of endothelial cells (ECs) and smooth muscle cell (SMCs) induced by ox-LDL and LOX-1. Angiogenesis (-): let-7g presumably inhibit the formation of plaque angiogenesis by TGF- $\beta$ signaling pathway, hence reduce the intraplaque hemorrhage. MMPs(-): It is predicted that let-7g could stabilize atherosclerosis plaque by increasing the ECM of fibrous cap and atherosclerosis plaque by down-regulating MMPs. But higher level of ECM might weaken the phenomenon of VSMCs migration from original site to fibrous cap, which is harmful to the stability of atherosclerosis plaque. Foam cells(-): It is predicted that let-7g reduce the number of foam cells through inhibiting the phagocytosis of macrophage induced by ox-LDL. 
MiRNAs have also emerged in regulating the complicated regulatory network during heart development [11]. Furthermore, recent reports suggest that miRNAs plays substantial roles in the development of atherosclerosis [12]. Various miRNAs have effects on controlling the integrity of vessels and the function of macrophage in every stage of atherosclerosis. The work from Chen's group suggested that miR-29b was up-regulated in vascular smooth muscle cells (VSMCs) with ox-LDL treatment, which might target on MMP2 leading to a reduction of VSMC migration [13]. Lovren and colleagues have shown that overexpressing miR-145 in VSMCs decreased the plaque size of atherosclerosis and favor plaque stability [14].

Let-7 family, belonging to an evolutionarily-conserved family of miRNAs, contains 12 members in mammal [15]. Let-7g is one of the best-characterized members of the Let-7 family. Like other miRNAs, let-7g appears to play a uniquely primary role in the pathogenesis of disease [16]. Frangogiannis et al. reviewed the relation between let-7g and endothelial cell function and proposed that let-7g induced preservation on vascular diseases [17] [18]. Let-7g is indicated to negatively regulate lectin-like oxidized LDL receptor-1 (LOX-1) through the intracellular $\mathrm{Ca}^{2+}$-activated protein kinase C-oxLDL-LOX-1-let-7g pathway [19]. Furthermore, let-7g has been found as an important modulator in the development of atherosclerosis over the last 5 years [12] [17]. The recent progression in let-7g might help to get insight into the potential effects of let- $7 \mathrm{~g}$ on regulating the progression of atherosclerosis-related diseases. In this review, we aim to discuss the current and potential roles of let-7g on the stabilization of atherosclerotic plaques.

\section{Data Sources}

We searched PubMed, Embase using terms "MicroRNAs/miRNAs/let-7" and "inflammation/macrophage" or "extracellular matrix/ECM/matrix" or "apoptosis" or "angiogenesis/neovascularization" for literatures we referred in this review.

\section{The Current and Potential Mechanisms of Let-7g on the Stabilizing of Atherosclerosis Plaque}

\subsection{Let-7g and Inflammation}

Atherosclerosis is considered as a chronic inflammatory disease due to the infiltration of immune cells into lesions and the production of pro-inflammatory cytokines [20], which contribute to the formation of vulnerable atherosclerotic plaques. Once infiltrated into the subendothelial space, some immune cells will unlimitedly take up lipids to continuously enlarge plaque [21]. The death of macrophage could induce plaque necrosis in advanced plaques, which may result in plaques instability and thus cause plaque rupture. Therefore, strategies to prevent macrophages apoptosis may stabilize the vulnerable plaques and reduce the life-threatening complications [21]. In addition, the interplays among immune cells, endothelial cells, platelets and smooth muscle cells facilitate the progres- 
sion of the atherosclerotic plaques [22] [23].

MicroRNAs are emerging as a new type of inflammatory regulators during atherosclerosis progression. Among them, let-7g was regarded to target inflammatory molecules. Let-7g, as an endogenous inhibitor of endothelial inflammatory activation, exerts a critical role in protecting endothelial cells [24] and stabling of atherosclerotic plaques. Let-7g leads to beneficial effects on EC through modulating TGF- $\beta$ signaling and SIRT-1 signaling (Figure 2) [24], which might contribute to the limitation of local inflammation of plaque and improve vascular thrombosis. In addition, overexpression of let- $7 \mathrm{~g}$ has resulted in the reduction of adhesion and migration of monocytes across endothelium [25].

Macrophages are important inflammation cells in the formation of vulnerable plaque. Anti-inflammatory M2 macrophages help to stabilize atherosclerotic plaques [26]. Our research found that the relative expression levels of let-7g in different macrophages were different. After macrophages phagocytized ox-LDL, the relative expression levels of let-7g changed. So, we hypothesized that let- $7 \mathrm{~g}$ has a regulatory effect on macrophages in atherosclerotic plaques, and the specific regulatory mechanisms need to be further studied. Currently, what is the role of Let-7g in macrophage polarization during atherosclerosis remains largely unknown. This could be a new field of research in atherosclerosis in the near future.

\subsection{Let-7g and Extracellular Matrix (ECM) or Collagen}

In addition to inflammation, the status of extracellular matrix (ECM) of arteries is germane to the stabilization of atherosclerotic plaques. Plaques with high coll-

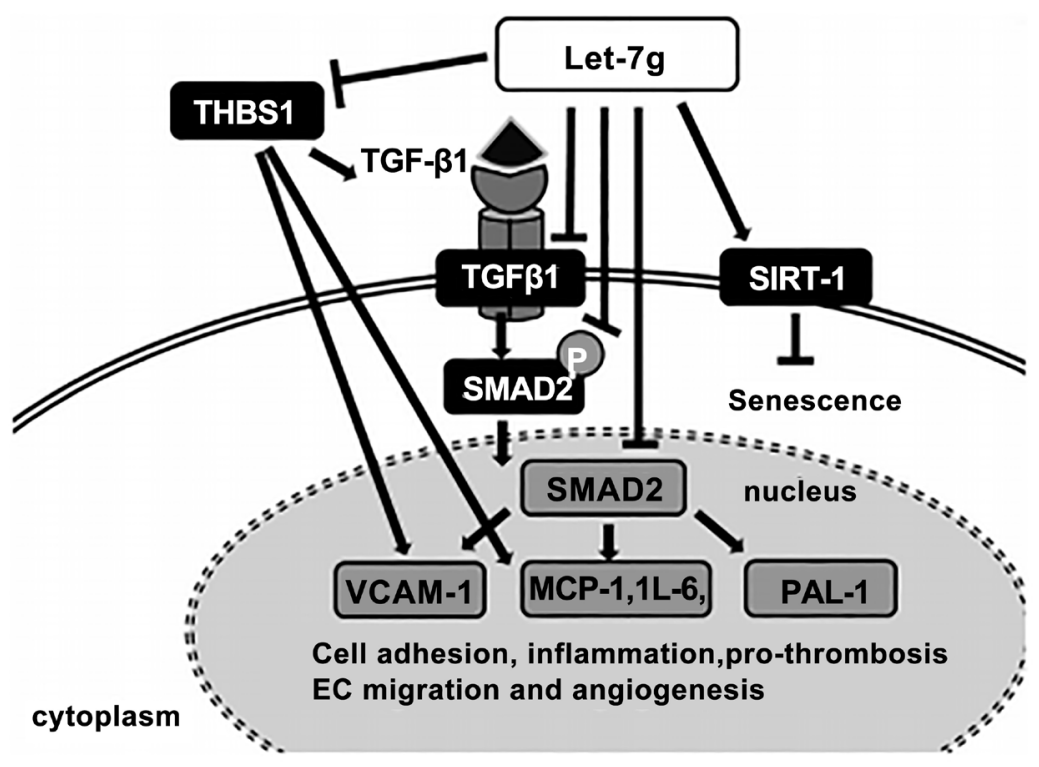

Figure 2. Schematic showed pleotropic effects of let-7g on endothelial functions. Let-7g leads to beneficial effects on EC through modulating TGF- $\beta$ signaling and SIRT-1 signaling, which might contribute to the limitation of local inflammation of plaque and improve vascular thrombosis. 
agen levels are more stable than plaques containing lower amounts of ECM protein [27]. The stabilization of atherosclerotic plaque depends on the content of ECM or collagen, the thickness of fibrous cap, the levels of proteases especially matrix metalloproteinases (MMPs) [1].

MMPs is one big family of calcium-dependent, zinc-containing endopeptidases and often produced by inflammatory cells, such as foam cells and macrophages, within atherosclerotic plaques. MMPs have strong ability to remodel the extracellular matrix components [28] [29] [30]. It could digest extracellular matrix, which is beneficial to immigration of VSMC from the original site to the fiber cap, reduce the thickness of fibrous cap, and thus promote plaque rupture [31]. Johnson et al. [32] showed that a specific MMP-12 inhibitor could retard the development of atherosclerosis and increase fibrosis within the plaque in an atherosclerotic mouse model. Also, it was found that patients with carotid atherosclerosis had markedly increased levels of MMP-7 in plasma, especially whom with newly diagnosed symptoms [33]. Silencing MMP-9 by RNA interference in the mice arteries had been shown to increase the thickness of the fibrous cap [34]. The secretion of MMPs, mainly MMP- 2 and MMP-9 by macrophages in plaque, has been associated with the vulnerable atherosclerotic plaque rupture [35]. Other numerous reports have shown that MMP-9 could induce monocyte/ macrophage differentiation, which resulted in progression and destabilization of atherosclerotic plaque [36] [37] [38].

Although MMPs have been considered as a negative player in atherosclerotic plaque formation, it could act positively in the maintenance of atherosclerotic plaque stability on other aspects. Some reports suggested that MMP-2, MMP-9, and MMP-14 promoted migration and proliferation of VSMCs, which could increase fibrous cap thickness and maintain plaque stability [3] [39]. Therefore, MMPs seem to act as double-edged sword in plaque stabilization due to their different characteristics.

At present, although little is known about the effect of let-7g on MMPs in atherosclerosis, let-7g has been shown to suppress the expression and activity of MMP-2/-9 in breast cancer [40], which indicates that let-7g may also contribute to the development of atherosclerotic plaque by regulating MMPs. Since MMPs might induce plaque rupture, it is possible that down-regulation of let-7g in susceptible individuals may increase MMPs effects and thus promote formation of unstable lesions [17]. In addition, different MMPs have differential effects on plaque stabilization. Which one is dominant becomes an issue of concern. The intriguing relationship between let-7g and MMPs on plaque stabilization is worth to further investigation.

\subsection{Let-7g and Apoptosis}

Apoptosis of endothelial cells (ECs) and VSMCs could also influence stability of plaque. OxLDL, usually expressed higher in hyperlipidemia patients, has the toxicity effect on vascular endothelial cells, induced EC apoptosis through mitochondrial and death receptor (Fas/FasL) apoptotic pathways [41], which is as- 
sociated with thin and incomplete of fibrous cap. VSMCs, the main structure of artery medium layer, also play a significant role on the formation of advanced stage of atherosclerosis. Apoptosis of VSMCs is harmful to the stability of atherosclerosis plaque [42]. Therefore, maintenance of VSMCs has potential to delay the progression of atherosclerosis [31].

It is well known that CASP3 is the major executioner caspase in apoptosis, and involves in cell cycle control, migration, and differentiation [43] [44]. Targeting CASP3 by hsa-let-7g significantly decreases apoptosis of endothelial cells induced by the ox-LDL. Hence, let-7g may have a significant influence on regulating ox-LDL-induced apoptosis and atherosclerosis [43] [45]. Report from Zhang group also suggested that overexpression of let-7g suppressed CASP3 expression and reduced apoptosis of ECs and the decreased levels of let-7g were found in apoptotic ECs elicited by ox-LDL [46]. As to VSMCs, observations from Ding group suggested that hsa-let-7g acts as a critical regulator of autophagy and apoptosis by modulating oxidized low density lipoprotein (lectionlike) receptor 1 (LOX-1, aka. OLR1). Hsa-let-7g can reduce SMC apoptosis through down-regulating cytochrome $\mathrm{c}$ and Smac/Diablo and up-regulating Bcl$\mathrm{xL}$ and Bcl-2 expression [47]. Moreover, let-7g can down-regulate LOX-1 expression in a dose-dependent and time-dependent manner. Together, these studies indicate let-7g can suppress apoptosis and promote cell survival by negatively regulating LOX-1 expression [48] [49] or other pathways (Figure 3).

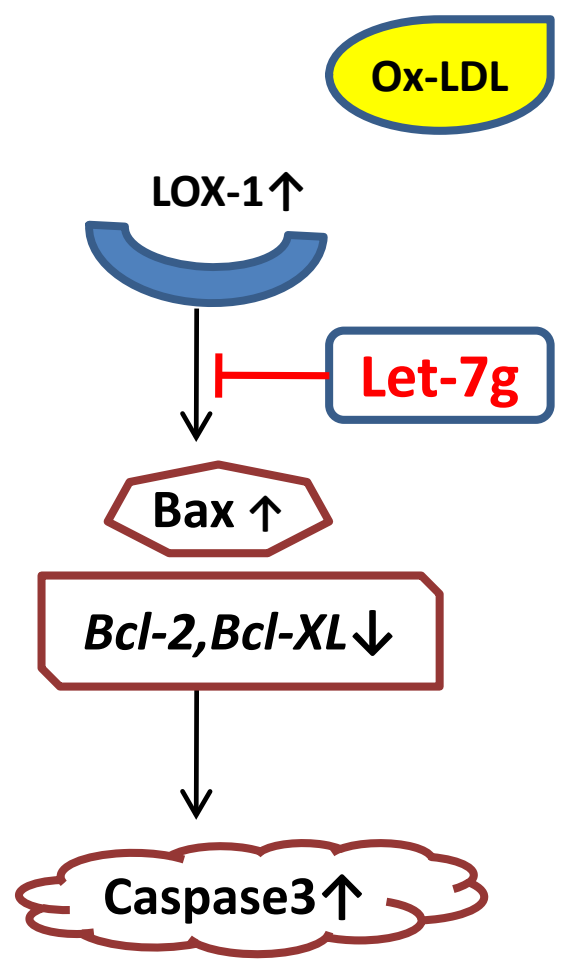

Figure 3. The effects of let-7g on inhibiting apoptosis of endothelial cells and vascular smooth muscular cells by negatively regulating LOX-1 expression or apoptotic molecules. 
Current data suggest that let-7g is an important regulator of apoptosis of ECs and SMCs. Let-7g could also regulate other cellular apoptosis contributed to atherosclerosis. Thus it might be a potential target for therapeutic intervention. Insights into the regulation of let- $7 \mathrm{~g}$ in atherosclerosis-related apoptosis might guide to the development of an alternative strategy by targeting let-7g in treatment of disease characterized by LOX-1 over-expression such as atherosclerosis [46].

\subsection{Let-7g and Intraplaque Ectopic Angiogenesis}

Plaque angiogenesis is a physiological response to the increased oxygen demand in the plaque, which has adverse effects on plaque vulnerability. Neovessels in plaques are usually fragility which allows for extravasation of lipoproteins. However, they can also promote the formation and enlargement of plaque lipid nucleus by letting red blood cells enter plaques, and results in plaque destabilization by increasing intraplaque hemorrhage and the infiltration of inflammatory cells [50] [51] [52]. Numerous studies have demonstrated that the co-existance of inflammatory and hypoxia in the plaque is a major stimulus for neovascularization [50] [51] [52] [53]. Indeed, it has been found that elevated oxygen tension could reduce hypoxia and inhibit the neovascularization of plaque and the progression lesion in mice [54]. Also, the inhibition of the lipid-driven inflammation or monocyte influx has similar results [55]. Therefore, atherosclerosis promotes the development of angiogenesis, which in return accelerates the progression of plaques. Therefore, the inhibition of intraplaque angiogenesis seems to be especially important to increase the stability of plaque. From this point, anti-angiogenic substances have been considered as apotential targeted therapy to stable plaques [17] [56].

Various miRNAs are associated with angiogenesis during different stages of atherosclerosis. According to published studies, let-7, miR-126, -143/145, -155, $-17-92$ and $-222 / 221$ could regulate angiogenesis [57] [58] [59] [60] [61]. Among them, the effects of let-7 on angiogenesis were firstly found by the knockdown of Dicer and Drosha in mouse which reduced let-7a, let-7b, let-7c, let-7f and let-7gmore than $30 \%$. On the other hand, the reduction of let-7a, let- $7 \mathrm{~b}$ and let- $7 \mathrm{f}$ has been verified to contribute to the sprouting of microvessels [62] [63] [64]. Recently, let-7g was shown to decrease angiogenesis and increase $\mathrm{EC}$ inflammation and monocyte adhesion via alterations in the TGF- $\beta$ signaling pathway. Liao's study suggested that venous injection of let-7g inhibitor into apolipoprotein E knockout mice increased the growth of vessels, the expression of PAI-1, and promoted the macrophage infiltration. At the same time, the up-regulations of TGF- $\beta$ downstream genes were detected in the carotid arteries, and suppression of the TGF- $\beta$ signaling by siRNAs could recover the angiogenesis [24]. Frangogiannis found that let-7g down-regulates expression of thrombospondin (TSP)-1, a matricellular protein with potent direct angiostatic properties [65] [66]. Thus, its impact on collateral circulation remains to be further investigated. These evidences indicate that let- $7 \mathrm{~g}$ plays important roles in plaque 
angiogenesis, but the underlie mechanism is complex and needs further studies.

\section{Conclusion}

Overall, let-7g has been suggested to contribute to a series of improvement in pathological process of atherosclerosis, which might benefit from preventing aggravation of atherosclerosis (Figure $1(B)$ ). Let-7g actions may represent a promising therapy for atherosclerotic disease [12]. But the implementation of miRNA let-7g-targeting strategies in vascular disease remains a daunting task. More robust in vivo experiment and careful analysis of the pathophysiologic pathways are needed to support the potential clinical application of let-7g-targeting strategies.

\section{Fund}

Funding for this study was provided by the National Natural Science Foundation of China (grant No. 81170173).

\section{References}

[1] Newby, A.C. (2007) Metalloproteinases and Vulnerable Atherosclerotic Plaques. Trends in Cardiovascular Medicine, 17, 253-258. https://doi.org/10.1016/j.tcm.2007.09.001

[2] Naghavi, M., Libb, P., Falk, E., Casscells, S.W., Litovsky, S., Rumberger, J., et al. (2003) From Vulnerable Plaque to Vulnerable Patient: A Call for New Definitions and Risk Assessment Strategies: Part II. Circulation, 108, 1772-1778. https://doi.org/10.1161/01.CIR.0000087481.55887.C9

[3] Liu, X.Q., Mao, Y., Wang, B., Lu, X.T., Bai, W.W., Sun, Y.Y., et al. (2014) Specific Matrix Metalloproteinases Play Different Roles in Intraplaque Angiogenesis and Plaque Instability in Rabbits. PLOS ONE, 9, e107851. https://doi.org/10.1371/journal.pone.0107851

[4] Virmani, R., Kolodgie, F.D., Burke, A.P., Finn, A.V., Gold, H.K., Tulenko, T.N., et al. (2005) Atherosclerotic Plaque Progression and Vulnerability to Rupture: Angiogenesis as a Source of Intraplaque Hemorrhage. Arteriosclerosis, Thrombosis, and Vascular Biology, 25, 2054-2061. https://doi.org/10.1161/01.ATV.0000178991.71605.18

[5] Kim, V.N. (2005) MicroRNA Biogenesis: Coordinated Cropping and Dicing. Nature Reviews Molecular Cell Biology, 6, 376-385. https://doi.org/10.1038/nrm1644

[6] Siddeek, B., Inoubli, L., Lakhdari, N., Rachel, P.B., Fussell, K.C., Schneider, S., et al. (2014) MicroRNAs as Potential Biomarkers in Diseases and Toxicology. Mutation Research: Genetic Toxicology and Environmental Mutagenesis, 764-765, 46-57. https://doi.org/10.1016/j.mrgentox.2014.01.010

[7] Bartel, D.P. (2004) MicroRNAs: Genomics, Biogenesis, Mechanism, and Function. Cell, 116, 281-297. https://doi.org/10.1016/S0092-8674(04)00045-5

[8] Fichtlscherer, S., De, R.S., Fox, H., Schwietz, T., Fischer, A., Liebetrau, C., et al. (2010) Circulating microRNAs in Patients with Coronary Artery Disease. Circulation Research, 107, 677-684. https://doi.org/10.1161/CIRCRESAHA.109.215566

[9] Vasa-Nicotera, M., Chen, H., Tucci, P., Yang, A.L., Saintigny, G., Menghini, R., et al. (2011) MiR-146a Is Modulated in Human Endothelial Cell with Aging. Atherosclerosis, 217, 326-330. https://doi.org/10.1016/j.atherosclerosis.2011.03.034 
[10] Kuppusamy, K.T., Jones, D.C., Sperber, H., Madan, A., Fischer, K.A., Rodriguez, M.L., et al. (2015) Let-7 Family of MicroRNA Is Required for Maturation and Adult-Like Metabolism in Stem Cell-Derived Cardiomyocytes. Proceedings of the National Academy of Sciences of the United States of America, 112, E2785-E2794. https://doi.org/10.1073/pnas.1424042112

[11] Kuppusamy, K.T., Sperber, H. and Ruohola-Baker, H. (2013) MicroRNA Regulation and Role in Stem Cell Maintenance, Cardiac Differentiation and Hypertrophy. Current Molecular Medicine, 13, 757-764. https://doi.org/10.2174/1566524011313050007

[12] Zampetaki, A. and Mayr, M. (2012) MicroRNAs in Vascular and Metabolic Disease. Circulation Research, 110, 508-522. https://doi.org/10.1161/CIRCRESAHA.111.247445

[13] Chen, K.C., Wang, Y.S., Hu, C.Y., Chang, W.C., Liao, Y.C., Dai, C.Y., et al. (2011) OxLDL Up-Regulates microRNA-29b, Leading to Epigenetic Modifications of MMP2/MMP-9 Genes: A Novel Mechanism for Cardiovascular Diseases. FASEB Journal, 25, 1718-1728. https://doi.org/10.1096/fj.10-174904

[14] Lovren, F., Pan, Y., Quan, A., Singh, K.K., Shukla, P.C., Gupta, N., et al. (2012) MicroRNA-145 Targeted Therapy Reduces Atherosclerosis. Circulation, 126, S81-S90. https://doi.org/10.1161/circulationaha.111.084186

[15] Thornton, J.E. and Gregory, R.I. (2012) How Does Lin28 let-7 Control Development and Disease? Trends in Cell Biology, 22, 474-482. https://doi.org/10.1016/j.tcb.2012.06.001

[16] Mendell, J.T. and Olson, E.N. (2012) MicroRNAs in Stress Signaling and Human Disease. Cell, 148, 1172-1187. https://doi.org/10.1016/j.cell.2012.02.005

[17] Frangogiannis, N.G. (2014) MicroRNAs and Endothelial Function: Many Challenges and Early Hopes for Clinical Applications. Journal of the American College of Cardiology, 63, 1695-1696. https://doi.org/10.1016/j.jacc.2013.10.056

[18] Bye, A., Rosjo, H., Nauman, J., Silva, G.J., Follestad, T., Omland, T., et al. (2016) Circulating microRNAs Predict Future Fatal Myocardial Infarction in Healthy Individuals-The HUNT Study. Journal of Molecular and Cellular Cardiology, 97, 162 168. https://doi.org/10.1016/j.yjmcc.2016.05.009

[19] Chen, K.C., Hsieh, I.C., Hsi, E., Wang, Y.S., Dai, C.Y., Chou, W.W., et al. (2011) Negative Feedback Regulation between microRNA let-7g and the oxLDL Receptor LOX-1. Journal of Cell Science, 124, 4115-4124. https://doi.org/10.1242/jcs.092767

[20] Libby, P., Lichtman, A.H. and Hansson, G.K. (2013) Immune Effector Mechanisms Implicated in Atherosclerosis: From Mice to Humans. Immunity, 38, 1092-1104. https://doi.org/10.1016/j.immuni.2013.06.009

[21] Karunakaran, D., Geoffrion, M., Wei, L., Gan, W., Richards, L., Shangari, P., et al. (2016) Targeting Macrophage Necroptosis for Therapeutic and Diagnostic Interventions in Atherosclerosis. Science Advances, 2, e1600224. https://doi.org/10.1126/sciadv.1600224

[22] Legein, B., Temmerman, L., Biessen, E.A. and Lutgens, E. (2013) Inflammation and Immune System Interactions in Atherosclerosis. Cellular and Molecular Life Sciences, 70, 3847-3869. https://doi.org/10.1007/s00018-013-1289-1

[23] Hansson, G.K. and Hermansson, A. (2011) The Immune System in Atherosclerosis. Nature Immunology, 12, 204-212. https://doi.org/10.1038/ni.2001

[24] Liao, Y.C., Wang, Y.S., Guo, Y.C., Lin, W.L., Chan, M.H. and Juo, S.H. (2014) Let-7g Improves Multiple Endothelial Functions through Targeting Transforming Growth Factor-Beta and SIRT-1 Signaling. Journal of the American College of Car- 
diology, 63, 1685-1694. https://doi.org/10.1016/j.jacc.2013.09.069

[25] Rom, S., Dykstra, H., Zuluaga-Ramire, V., Reichenbach, N.L. and Persidsky, Y. (2015) MiR-98 and let-7g Protect the Blood-Brain Barrier under Neuroinflammatory Conditions. Journal of Cerebral Blood Flow \& Metabolism, 35, 1957-1965. https://doi.org/10.1038/jcbfm.2015.154

[26] Medbury, H.J., Williams, H. and Fletcher, J.P. (2014) Clinical Significance of Macrophage Phenotypes in Cardiovascular Disease. Clinical and Translational Medicine, 3, 63. https://doi.org/10.1186/s40169-014-0042-1

[27] Hofnagel, O., Robenek, H. and Cathepsin, K. (2009) Boon or Bale for Atherosclerotic Plaque Stability? Cardiovascular Research, 81, 242-243. https://doi.org/10.1093/cvr/cvn343

[28] Vacek, T.P., Rehman, S., Neamtu, D., Yu, S., Givimani, S. and Tyagi, S.C. (2015) Matrix Metalloproteinases in Atherosclerosis: Role of Nitric Oxide, Hydrogen Sulfide, Homocysteine, and Polymorphisms. Vascular Health and Risk Management, 11, 173-183. https://doi.org/10.2147/VHRM.S68415

[29] Austin, K.M., Covic, L. and Kuliopulos, A. (2013) Matrix Metalloproteases and PAR1 Activation. Blood, 121, 431-439. https://doi.org/10.1182/blood-2012-09-355958

[30] Heo, S.H., Cho, C.H., Kim, H.O., Jo, Y.H., Yoon, K.S., Lee, J.H., et al. (2011) Plaque Rupture Is a Determinant of Vascular Events in Carotid Artery Atherosclerotic Disease: Involvement of Matrix Metalloproteinases 2 and 9. Journal of Clinical Neurology, 7, 69-76. https://doi.org/10.3988/jcn.2011.7.2.69

[31] Allahverdian, S., ChehroudiM, A.C., McManus, B.M., Abraham, T. and Francis, G.A. (2014) Contribution of Intimal Smooth Muscle Cells to Cholesterol Accumulation and Macrophage-Like Cells in Human Atherosclerosis. Circulation, 129, 1551 1559. https://doi.org/10.1161/CIRCULATIONAHA.113.005015

[32] Johnson, J.L., Devel, L., Czarny, B., George, S.J., Jackson, C.L., Rogakos, V., et al. (2011) A Selective Matrix Metalloproteinase-12 Inhibitor Retards Atherosclerotic plaque Development in Apolipoprotein E-Knockout Mice. Arteriosclerosis, Thrombosis, and Vascular Biology, 31, 528-535. https://doi.org/10.1161/ATVBAHA.110.219147

[33] Abbas, A., Aukrust, P., Russell, D., Krohg-Sorensen, K., Almas, T., Bundgaard, D., et al. (2014) Matrix Metalloproteinase 7 Is Associated with Symptomatic Lesions and Adverse Events in Patients with Carotid Atherosclerosis. PLoS ONE, 9, e84935. https://doi.org/10.1371/journal.pone.0084935

[34] Jin, Z.X., Xiong, Q., Jia, F., Sun, C.L., Zhu, H.T. and Ke, F.S. (2015) Investigation of RNA Interference Suppression of Matrix Metalloproteinase-9 in Mouse Model of Atherosclerosis. International Journal of Clinical and Experimental Medicine, 8, 5272-5278.

[35] Libby, P. (2001) What Have We Learned about the Biology of Atherosclerosis? The Role of Inflammation. American Journal of Cardiology, 88, 3J-6J. https://doi.org/10.1016/s0002-9149(01)01879-3

[36] Yoon, Y.W., Kwon, H.M., Hwang, K.C., Choi, E.Y., Hong, B.K., Kim, D., et al. (2005) Upstream Regulation of Matrix Metalloproteinase by EMMPRIN. Extracellular Matrix Metalloproteinase Inducer in Advanced Atherosclerotic Plaque. Atherosclerosis, 180, 37-44. https://doi.org/10.1016/j.atherosclerosis.2004.11.021

[37] Newby, A.C. (2008) Metalloproteinase Expression in Monocytes and Macrophages and Its Relationship to Atherosclerotic Plaque Instability. Arteriosclerosis, Thrombosis, and Vascular Biology, 28, 2108-2114. https://doi.org/10.1161/ATVBAHA.108.173898 
[38] Cimmino, G., Ragni, M., Cirillo, P., Petrillo, G., Loffredo, F., Chiariello, M., et al. (2013) C-Reactive Protein Induces Expression of Matrix Metalloproteinase-9: A Possible Link between Inflammation and Plaque Rupture. International Journal of Cardiology, 168, 981-986. https://doi.org/10.1016/j.ijcard.2012.10.040

[39] Benjamin, M.M. and Khalil, R.A. (2012) Matrix Metalloproteinase Inhibitors as Investigative Tools in the Pathogenesis and Management of Vascular Disease. Exs, 103, 209-279. https://doi.org/10.1007/978-3-0348-0364-9_7

[40] Qian, P., Zuo, Z., Wu, Z., Meng, X., Li, G., Wu, Z., et al. (2011) Pivotal Role of Reduced Let-7g Expression in Breast Cancer Invasion and Metastasis. Cancer Research, 71, 6463-6474. https://doi.org/10.1158/0008-5472.CAN-11-1322

[41] Colles, S.M., Maxson, J.M., Carlson, S.G. and Chisolm, G.M. (2001) Oxidized LDL-Induced Injury and Apoptosis in Atherosclerosis. Potential Roles for Oxysterols. Trends in Cardiovascular Medicine, 11, 131-138. https://doi.org/10.1016/S1050-1738(01)00106-2

[42] Mallat, Z. and Tedgui, A. (2000) Apoptosis in the Vasculature: Mechanisms and Functional Importance. British Journal of Pharmacology, 130, 947-962.

https://doi.org/10.1038/sj.bjp.0703407

[43] Schwerk, C. and Schulze-Osthoff, K. (2003) Non-Apoptotic Functions of Caspases in Cellular Proliferation and Differentiation. Biochemical Pharmacology, 66, 14531458. https://doi.org/10.1016/S0006-2952(03)00497-0

[44] Acarin, L., Villapol, S., Faiz, M., Rohn, T.T., Castellano, B. and Gonzalez, B. (2007) Caspase-3 Activation in Astrocytes Following Postnatal Excitotoxic Damage Correlates with Cytoskeletal Remodeling but Not with Cell Death or Proliferation. Glia, 55, 954-965. https://doi.org/10.1002/glia.20518

[45] Nhan, T.Q., Liles, W.C. and Schwartz, S.M. (2006) Physiological Functions of Caspases beyond Cell Death. American Journal of Pathology, 169, 729-737. https://doi.org/10.2353/ajpath.2006.060105

[46] Zhang, Y., Chen, N., Zhang, J. and Tong, Y. (2013) Hsa-Let-7g miRNA Targets Caspase- 3 and Inhibits the Apoptosis Induced by OX-LDL in Endothelial Cells. International Journal of Molecular Sciences, 14, 22708-22720. https://doi.org/10.3390/ijms141122708

[47] Ding, Z., Wang, X., Khaidakov, M., Liu, S. and Mehta, J.L. (2012) MicroRNA Hsa-Let-7g Targets Lectin-Like Oxidized Low-Density Lipoprotein Receptor-1 Expression and Inhibits Apoptosis in Human Smooth Muscle Cells. Experimental Biology and Medicine, 237, 1093-1100. https://doi.org/10.1258/ebm.2012.012082

[48] Ding, Z., Wang, X., Schnackenberg, L., Khaidakov, M., Liu, S., Singla, S., et al. (2013) Regulation of Autophagy and Apoptosis in Response to OX-LDL in Vascular Smooth Muscle Cells, and the Modulatory Effects of the microRNA Hsa-Let-7g. International Journal of Cardiology, 168, 1378-1385.

https://doi.org/10.1016/j.ijcard.2012.12.045

[49] Wu, K., Yang, Y., Zhao, J. and Zhao, S. (2016) BAG3-Mediated miRNA Let-7g and Let-7i Inhibit Proliferation and Enhance Apoptosis of Human Esophageal Carcinoma Cells by Targeting the Drug Transporter ABCC10. Cancer Letters, 371, 125-133. https://doi.org/10.1016/j.canlet.2015.11.031

[50] Sluimer, J.C., Gasc, J.M., van Wanroij, J.L., Kisters, N., Groeneweg, M., Sollewijn Gelpke, M.D., et al. (2008) Hypoxia, Hypoxia-Inducible Transcription Factor, and Macrophages in Human Atherosclerotic Plaques Are Correlated with Intraplaque Angiogenesis. Journal of the American College of Cardiology, 51, 1258-1265. https://doi.org/10.1016/j.jacc.2007.12.025

[51] Lin, H.L., Zhang, L., Liu, C.X., Xu, X.S., Tang, M.X., Lv, H.X., et al. (2010) Hae- 
min-Enhanced Expression of Haem Oxygenase-1 Stabilizes Erythrocyte-Induced Vulnerable Atherosclerotic Plaques. British Journal of Pharmacology, 160, 1484-1495. https://doi.org/10.1111/j.1476-5381.2010.00799.x

[52] Lin, H.L., Xu, X.S., Lu, H.X., Zhang, L., Li, C.J., Tang, M.X., et al. (2007) Pathological Mechanisms and Dose Dependency of Erythrocyte-Induced Vulnerability of Atherosclerotic Plaques. Journal of Molecular and Cellular Cardiology, 43, 272-280. https://doi.org/10.1016/j.yjmcc.2007.05.023

[53] de Vries, M.R. and Quax, P.H. (2016) Plaque Angiogenesis and Its Relation to Inflammation and Atherosclerotic Plaque Destabilization. Current Opinion in Lipidology, 27, 499-506. https://doi.org/10.1097/MOL.0000000000000339

[54] Marsch, E., Theelen, T.L., Demandt, J.A., Jeurissen, M., van Gink, M., Verjans, R., et al. (2014) Reversal of Hypoxia in Murine Atherosclerosis Prevents Necrotic Core Expansion by Enhancing Efferocytosis. Arteriosclerosis, Thrombosis, and Vascular Biology, 34, 2545-2553. https://doi.org/10.1161/ATVBAHA.114.304023

[55] Bot, I., Jukema, J.W., Lankhuizen, I.M., van Berkel, T.J. and Biessen, E.A. (2011) Atorvastatin Inhibits Plaque Development and Adventitial Neovascularization in ApoE Deficient Mice Independent of Plasma Cholesterol Levels. Atherosclerosis, 214, 295-300. https://doi.org/10.1016/j.atherosclerosis.2010.11.008

[56] Michel, J.B., Virmani, R., Arbustini, E. and Pasterkamp, G. (2011) Intraplaque Haemorrhages as the Trigger of Plaque Vulnerability. European Heart Journal, 32, 1977-1985. https://doi.org/10.1093/eurheartj/ehr054

[57] Madrigal-Matute, J., Rotllan, N., Aranda, J.F. and Fernandez-Hernando, C. (2013) MicroRNAs and Atherosclerosis. Current Atherosclerosis Reports, 15, 322. https://doi.org/10.1007/s11883-013-0322-Z

[58] Tabuchi, T., Satoh, M., Itoh, T. and Nakamura, M. (2012) MicroRNA-34a Regulates the Longevity-Associated Protein SIRT1 in Coronary Artery Disease: Effect of Statins on SIRT1 and microRNA-34a Expression. Clinical Science, 123, 161-171. https://doi.org/10.1042/CS20110563

[59] Tan, K.S., Armugam, A., Sepramaniam, S., Lim, K.Y., Setyowati, K.D., Wang, C.W., et al. (2009) Expression Profile of MicroRNAs in Young Stroke Patients. PLoS ONE, 4, e7689. https://doi.org/10.1371/journal.pone.0007689

[60] Rippe, C., Blimline, M., Magerko, K.A., Lawson, B.R., LaRocca, T.J., Donato, A.J., et al. (2012) MicroRNA Changes in Human Arterial Endothelial Cells with Senescence: Relation to Apoptosis, eNOS and Inflammation. Experimental Gerontology, 47, 45-51. https://doi.org/10.1016/j.exger.2011.10.004

[61] Santulli, G. (2015) MicroRNAs Distinctively Regulate Vascular Smooth Muscle and Endothelial Cells: Functional Implications in Angiogenesis, Atherosclerosis, and In-Stent Restenosis. Advances in Experimental Medicine and Biology, 887, 53-77. https://doi.org/10.1007/978-3-319-22380-3_4

[62] Kuehbacher, A., Urbich, C., Zeiher, A.M. and Dimmeler, S. (2007) Role of Dicer and Drosha for Endothelial microRNA Expression and Angiogenesis. Circulation Research, 101, 59-68. https://doi.org/10.1161/CIRCRESAHA.107.153916

[63] Suarez, Y., Fernandez-Hernando, C., Pober, J.S. and Sessa, W.C. (2007) Dicer Dependent microRNAs Regulate Gene Expression and Functions in Human Endothelial cells. Circulation Research, 100, 1164-1173. https://doi.org/10.1161/01.RES.0000265065.26744.17

[64] Otsuka, M., Zheng, M., Hayashi, M., Lee, J.D., Yoshino, O., Lin, S., et al. (2008) Impaired microRNA Processing Causes Corpus Luteum Insufficiency and Infertility in Mice. Journal of Clinical Investigation, 118, 1944-1954.

https://doi.org/10.1172/JCI33680 
[65] Frangogiannis, N.G. (2012) Matricellular Proteins in Cardiac Adaptation and Disease. Physiological Reviews, 92, 635-688.

https://doi.org/10.1152/physrev.00008.2011

[66] Gonzalez-Quesada, C., Cavalera, M., Biernacka, A., Kong, P., Lee, D.W., Saxena, A., et al. (2013) Thrombospondin-1 Induction in the Diabetic Myocardium Stabilizes the Cardiac Matrix in Addition to Promoting Vascular Rarefaction through Angiopoietin-2 Upregulation. Circulation Research, 113, 1331-1344.

https://doi.org/10.1161/CIRCRESAHA.113.302593

Submit or recommend next manuscript to SCIRP and we will provide best service for you:

Accepting pre-submission inquiries through Email, Facebook, LinkedIn, Twitter, etc. A wide selection of journals (inclusive of 9 subjects, more than 200 journals)

Providing 24-hour high-quality service

User-friendly online submission system

Fair and swift peer-review system

Efficient typesetting and proofreading procedure

Display of the result of downloads and visits, as well as the number of cited articles Maximum dissemination of your research work

Submit your manuscript at: http://papersubmission.scirp.org/

Or contact wjcd@scirp.org 